\title{
The Role of Angiotensin II in Parietal Epithelial Cell Proliferation and Crescent Formation in Glomerular Diseases
}

\author{
Paola Rizzo, ${ }^{*}$ Rubina Novelli, Cinzia Rota, ${ }^{*}$ Elena Gagliardini, ${ }^{*}$ Barbara Ruggiero, ${ }^{*}$ Daniela Rottoli, Ariela Benigni, and \\ Giuseppe Remuzzi* ${ }^{\dagger \ddagger}$
}

\begin{abstract}
From the IRCCS-Istituto di Ricerche Farmacologiche Mario Negri,* Centro Anna Maria Astori, Science and Technology Park Kilometro Rosso, Bergamo; the Unit of Nephrology, ${ }^{\dagger}$ Azienda Socio-Sanitaria Territoriale Papa Giovanni XXIII, Bergamo; and the Department of Biomedical and Clinical Sciences, ${ }^{\ddagger}$ University of Milan, Milan, Italy
\end{abstract}

\author{
Accepted for publication \\ July 6, 2017. \\ Address correspondence to \\ Ariela Benigni, Ph.D., \\ IRCCS-Istituto di Ricerche \\ Farmacologiche Mario Negri, \\ Centro Anna Maria Astori, \\ Science and Technology Park \\ Kilometro Rosso, Via \\ Stezzano 87, 24126 Bergamo, \\ Italy. E-mail: ariela.benigni@ \\ marionegri.it.
}

\begin{abstract}
Crescentic glomerulonephritis (GN) is a devastating disease with rapidly progressive deterioration in kidney function, which, histologically, manifests as crescent formation in most glomeruli. We previously found that crescents derive from the aberrant proliferation and migration of parietal epithelial cells (PECs)/progenitor cells, and that the angiotensin (ang) II/ang II type-1 ( $\mathrm{AT}_{1}$ ) receptor pathway may participate, together with the stromal cell-derived factor-1 (SDF-1)/C-X-C chemokine receptor 4 axis, in the development of those lesions. Herein, we elucidated sequential events and cellular and molecular interactions occurring during crescentic lesion onset and evolution. By analyzing kidney biopsy specimens of patients with extracapillary GN, divided according to the grade of glomerular lesions, we found that the accumulation of macrophages expressing matrix metalloproteinase- 12 started manifesting in glomeruli affected by early-stage lesions, whereas $\mathrm{AT}_{1}$ receptor expression could not be detected. In glomeruli with advanced lesions, $\mathrm{AT}_{1}$ receptor expression increased markedly, and the up-regulation of SDF-1, and its receptor C-X-C chemokine receptor 7, was documented on podocytes and PECs, respectively. In vitro studies were instrumental to demonstrating the role of ang II in inducing podocyte SDF-1 production, which ultimately activates PECs. The present findings support the possibility that angiotensin-converting enzyme inhibitor treatment might limit PEC activation and reduce the frequency and extension of crescents in extracapillary GN. (Am J Pathol 2017, 187: 2441-2450; http:// dx.doi.org/10.1016/j.ajpath.2017.07.004)
\end{abstract}

Glomerular crescentic lesions are the result of a pathologic accumulation of proliferating cells between the Bowman capsule and the capillary tuft. Crescentic lesions are found in glomeruli of patients affected by rapidly progressive glomerulonephritis (GN), a clinical syndrome characterized by rapid loss of renal function, often accompanied by hypertension, macroscopic hematuria, and proteinuria. ${ }^{1}$ Three types of crescentic GN are currently recognized: anti-glomerular basement membrane (GBM) GN, immune complex-mediated GN, and antineutrophil cytoplasm antibody-associated vasculitis, which is the most common form of crescentic GN, accounting for $>60 \%$ of all cases. $^{1-3}$ Identifying cellular components of crescents, and their pathogenic role, has attracted scientists' interest for decades. Early studies revealed that macrophages play a crucial role in the pathogenesis of crescentic GN, stimulating self-recruitment and promoting the proliferation of epithelial cells through the release of proinflammatory cytokines. ${ }^{4,5}$ It later became clear that crescentic lesions are composed of a mixture of cell types, including parietal epithelial cells (PECs), myofibroblasts, and

\footnotetext{
Supported byFondazione Aiuti per la Ricerca sulle Malattie Rare, Bergamo, Italy, fellowships (P.R. and R.N.); and the European Union's Seventh Framework programme HEALTHF4-2012-305436 (Project STELLAR).

P.R. and R.N. contributed equally to this work.

Disclosures: None declared.
} 
macrophages, ${ }^{6-8}$ with the latter predominating when a rupture of the Bowman capsule occurs. ${ }^{6,9,10}$ It has also been suggested that podocytes could contribute to crescent formation. ${ }^{11-15}$ However, given their terminally differentiated phenotype and the limited ability to migrate and proliferate, ${ }^{16}$ podocytes cannot be considered as major constituents of multilayered hyperplastic lesions. ${ }^{15,17}$ During the past decade, all evidence from human and animal experiments has pointed to PECs playing a key role in the pathogenesis of different glomerular diseases. ${ }^{18,19}$ In physiological conditions, a subset of PECs serves as a reservoir of renal progenitors, possibly contributing to podocyte turnover. ${ }^{20}$ However, in the presence of extensive glomerular damage, these cells can respond with exuberant proliferation and/or migration from the Bowman capsule toward the glomerular tuft. ${ }^{15,21-25}$

While looking for mediators responsible for the dysregulated response of renal progenitors in proliferative diseases, we previously suggested that the immune-related chemokine stromal cell-derived factor-1 (SDF-1)/C-X-C chemokine receptor (CXCR) 4 axis, ${ }^{22}$ known to be crucial for cell migration and proliferation, ${ }^{26,27}$ might play a role. In human and rat crescentic GN, marked SDF-1 and CXCR4 up-regulation in podocytes and PECs, respectively, suggested that SDF-1 produced by podocytes might trigger $\mathrm{PEC} /$ progenitor cell activation via CXCR4, leading to the formation of crescentic lesions. ${ }^{22}$ In accordance with evidence showing that angiotensin (ang) II can promote cell proliferation and migration through the ang II type- $1\left(\mathrm{AT}_{1}\right)$ receptor, ${ }^{28}$ we also found that $\mathrm{AT}_{1}$ receptor expression was up-regulated in patients' parietal progenitor cells and within hyperplastic lesions, ${ }^{22}$ and treatment with an angiotensinconverting enzyme (ACE) inhibitor significantly reduced the frequency and extent of crescents in an experimental model of severe proliferative disease. ${ }^{21}$ Moreover, a patient with antineutrophil cytoplasm antibody-positive extracapillary GN who displayed regression of crescentic lesions and reduction of $\mathrm{AT}_{1}$ receptor expression in renal progenitors in a repeated biopsy after ACE inhibitor treatment ${ }^{22}$ was also informative in this context.

Although our previous findings ${ }^{21,22}$ have allowed us to lay the groundwork for understanding the formation of crescents, what is still missing in the literature is an accurate analysis of the sequential events occurring during the onset and progression of glomerular hyperplastic lesions. As multiple biopsies at different time points cannot be performed in humans, herein we sought to outline a step-bystep sequence of crescent evolution by performing a serial section analysis of glomeruli displaying morphologically different degrees of lesions, and to investigate the cellular and molecular mediators involved. For this purpose, we analyzed kidney biopsy specimens from patients with extracapillary GN to i) evaluate the presence and extent of breaks affecting both the GBM and Bowman capsule, which may lead to macrophage infiltration in the Bowman space; ii) define at what stage of crescentic lesion evolution the accumulation of infiltrating macrophages expressing matrix metalloproteinase-12 (MMP-12) occurs, and how it was associated with $\mathrm{AT}_{1}$ receptor up-regulation; and iii) investigate whether the glomerular expression of the chemokine SDF-1 and its receptors CXCR4 and CXCR7 was modulated. Then, functional co-culture in vitro studies allowed us to dissect cellular and molecular mechanisms underlying the cross talk between progenitor cells and podocytes, crucial to hyperplastic lesion onset and progression.

\section{Materials and Methods}

\section{Patients}

Ten patients with extracapillary GN from the archives of the Unit of Nephrology, Azienda Socio-Sanitaria Territoriale Papa Giovanni XXIII (Bergamo, Italy), were enrolled in the study. All kidney biopsy specimens had originally been obtained to diagnose renal disease. Written informed consent was obtained from all patients. Demographic, clinical, and hematochemical parameters at the time of biopsy were retrieved from the hospital database. In addition to specimens from patients with extracapillary GN, renal biopsy specimens from an uninvolved portion of kidney collected from tumor nephrectomy specimens were obtained from 10 patients and used as controls.

\section{Quantification of Glomerular Crescents}

Renal biopsy specimens of patients with extracapillary GN were fixed in Duboscq-Brazil and embedded in paraffin. Sections (2 $\mu \mathrm{m}$ thick) were stained with periodic acid-Schiff reagent and observed by light microscopy (BH2-RFCA; Olympus, Melville, NY). All of the glomeruli present in renal biopsy specimens were examined for each patient, and the extent of crescents was expressed by giving a score from 0 to 4 , related to the percentage of the glomerular tuft that was occupied by the lesions $(0$, no lesions; 1 , lesions affecting $<25 \% ; 2, \geq 25 \%$ to $<50 \%$; 3 , $\geq 50 \%$ to $<75 \% ; 4, \geq 75 \%$ to $100 \%$ of the glomerulus). The index of crescents, which considers both the number and the extent of the lesions, was calculated by using the following formula: Index $=\left(1 \times n_{1}\right)+\left(2 \times n_{2}\right)+\left(3 \times n_{3}\right)+(4 \times$ $\left.\mathrm{n}_{4}\right) / \mathrm{n}_{0}+\mathrm{n}_{1}+\mathrm{n}_{2}+\mathrm{n}_{3}+\mathrm{n}_{4}$, where $\mathrm{n}_{\mathrm{x}}$ is the number of glomeruli for each percentage of crescent extension.

\section{Evaluation of the Integrity of the GBM and Bowman Capsule}

To detect basal membranes and to evaluate the integrity of the GBM and Bowman capsule, Duboscq-Brazil-fixed and paraffin-embedded kidney sections from patients with extracapillary GN and from normal kidneys were stained with silver methenamine reagents (Bio Optica, Milan, Italy) and observed by light microscopy (ApoTome AxioImager Z2; Zeiss, Jena, Germany). All of the glomeruli present in 
Table 1 Demographic, Clinical, and Histopathological Characteristics of Patient Populations

\begin{tabular}{lc}
\hline Characteristics & Value $(n=10)$ \\
\hline Age in years, range & $20-81$ \\
Male:female ratio & $2: 8$ \\
Clinical parameters & \\
$\quad$ Systolic blood pressure, mmHg & $135.4 \pm 14.4$ \\
$\quad$ Diastolic blood pressure, mmHg & $80.2 \pm 8.4$ \\
Laboratory parameters & \\
$\quad$ Proteinuria, g/day & $5.2 \pm 2.2$ \\
$\quad$ Serum creatinine, mg/dL & $3.4 \pm 2.6$ \\
$\quad$ Hematuria, 1:0 & $9: 1$ \\
Diagnosis, $n$ & \\
$\quad$ Anti-GBM antibody & $1^{*}$ \\
$\quad$ glomerulonephritis (type I) & \\
$\quad$ ANCA-associated or pauci-immune & $4^{\dagger}$ \\
$\quad$ vasculitis (type II) & \\
Immune-complex-mediated & $5^{\ddagger}$ \\
$\quad$ glomerular disease (type III) & \\
Histopathological findings & \\
$\quad$ Index of crescents, score 0-4 & \\
$\quad$ Glomeruli with extracapillary proliferation, \% & $56.0 \pm 35.3$ \\
$\quad$ Glomeruli with global sclerosis, \% & $21.4 \pm 20.3$ \\
\hline
\end{tabular}

Data are expressed as means \pm SD.

${ }^{*}$ Anti-GBM antibodies with associated p-ANCA antibodies.

'p-ANCA, $n=3$; c-ANCA, $n=1$.

${ }^{\ddagger}$ IgA nephropathy, $n=1$; lupus nephritis, $n=3$; immune complex-mediated glomerulonephritis secondary to systemic disease, $n=1$.

ANCA, antineutrophil cytoplasm antibody; C, cytoplasmic; GBM, glomerular basement membrane; $p$, perinuclear.

renal biopsy specimens were examined for each patient. The extent of Bowman capsule rupture was expressed by giving a score from 0 to 4 related to the percentage of the capsule affected by gaps ( 0 , intact Bowman capsule; 1, rupture affecting $<25 \% ; 2,25 \%$ to $50 \% ; 3,50 \%$ to $75 \% ; 4,75 \%$ to $100 \%$ of the Bowman capsule).

\section{Electron Microscopy Analysis}

Biopsy specimens were fixed with $2.5 \%$ glutaraldehyde in $0.1 \mathrm{~mol} / \mathrm{L}$ cacodylate buffer ( $\mathrm{pH} 7.4$ ) for 4 hours at $4^{\circ} \mathrm{C}$ and washed in cacodylate buffer. Kidney fragments were then postfixed in $1 \%$ osmium tetroxide for 1 hour, dehydrated through ascending grades of alcohol, and embedded in Epon resin (Electron Microscopy Science, Hatfield, PA). Ultrathin sections (60 to $100 \mathrm{~nm}$ thick) were cut on an EM UC7 ultramicrotome (Leica Microsystems, Mannheim, Germany), stained with uranyl acetate and lead citrate, and examined with transmission electron microscopy (Morgagni 268D; Philips, Brno, Czech Republic).

\section{Immunoperoxidase}

CD68, MMP-12, and $\mathrm{AT}_{1}$ receptor expression was analyzed by immunoperoxidase staining in human biopsy specimens. Duboscq-Brazil-fixed, paraffin-embedded kidney sections were deparaffinized, rehydrated, and incubated for 5 minutes with peroxidazed1 (Biocare Medical, Concord, CA) to quench endogenous peroxidases. Antigen retrieval was performed using a Decloaking chamber (DCNxGen 220V; Biocare Medical) for $15 \mathrm{mi}$ nutes at $110^{\circ} \mathrm{C}$ with DIVA decloaker buffer (Biocare Medical). After blocking for 5 minutes with Background Punisher (Biocare Medical), sections were incubated with mouse anti-CD68 (1:100; DakoCytomation, Glostrup, Denmark) or rabbit anti-AT ${ }_{1}$ receptor primary antibodies (1:25; Santa Cruz Biotechnology, Dallas, TX), followed by MACH4 HRP-Polymer kit (Biocare Medical) and diaminobenzidine (Biocare Medical) substrate solution. For
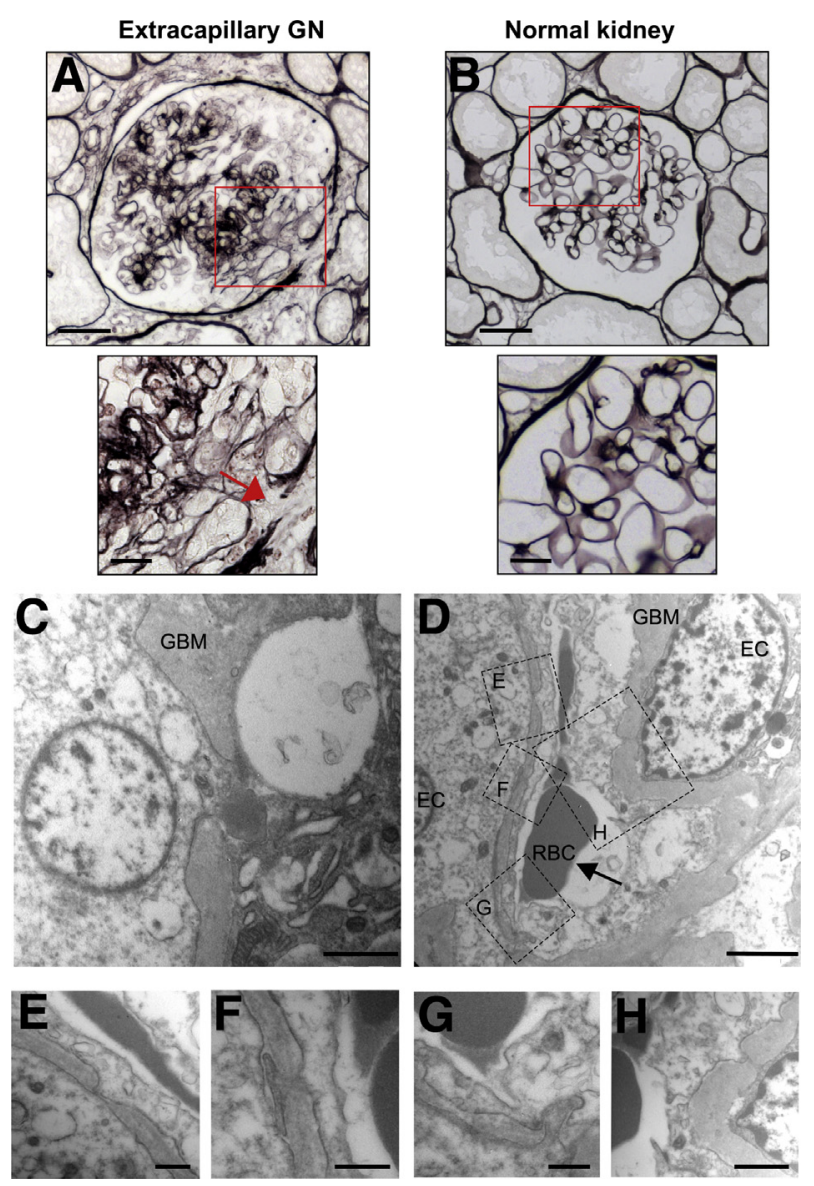

Figure 1 Histological and ultrastructural analysis of Bowman capsule and glomerular basement membrane (GBM). A: Top panel: Silver methenamine-stained renal biopsy specimen from a patient with extracapillary glomerulonephritis (GN). Bottom panel: A break in the Bowman capsule (arrow) and several irregularities and wrinkling along the GBM. B: Uninvolved portion of renal tissue removed because of renal cell carcinoma, used as age-matched control, displays glomeruli with intact Bowman capsule and linear GBM. Boxed areas in $\mathbf{A}$ and $\mathbf{B}$ are shown below at higher magnification. C: Representative transmission electron micrograph showing a rupture in the GBM. D: At the ultrastructural level, red blood cells (RBCs; arrow) were found outside of glomerular capillaries in the proximity of multiple GBM discontinuities. E-H: Higher magnification of the dashed boxes in $\mathbf{D}$, showing GBM discontinuities and gaps. Scale bars: $50 \mu \mathrm{m}$ (A and $\mathbf{B}$, top panels); $20 \mu \mathrm{m}$ (A and $\mathbf{B}$, bottom panels); $2000 \mathrm{~nm}$ (C and $\mathbf{D}$ ); $500 \mathrm{~nm}(\mathbf{E}-\mathbf{G}) ; 1000 \mathrm{~nm}$ (H). EC, endothelial cell. 


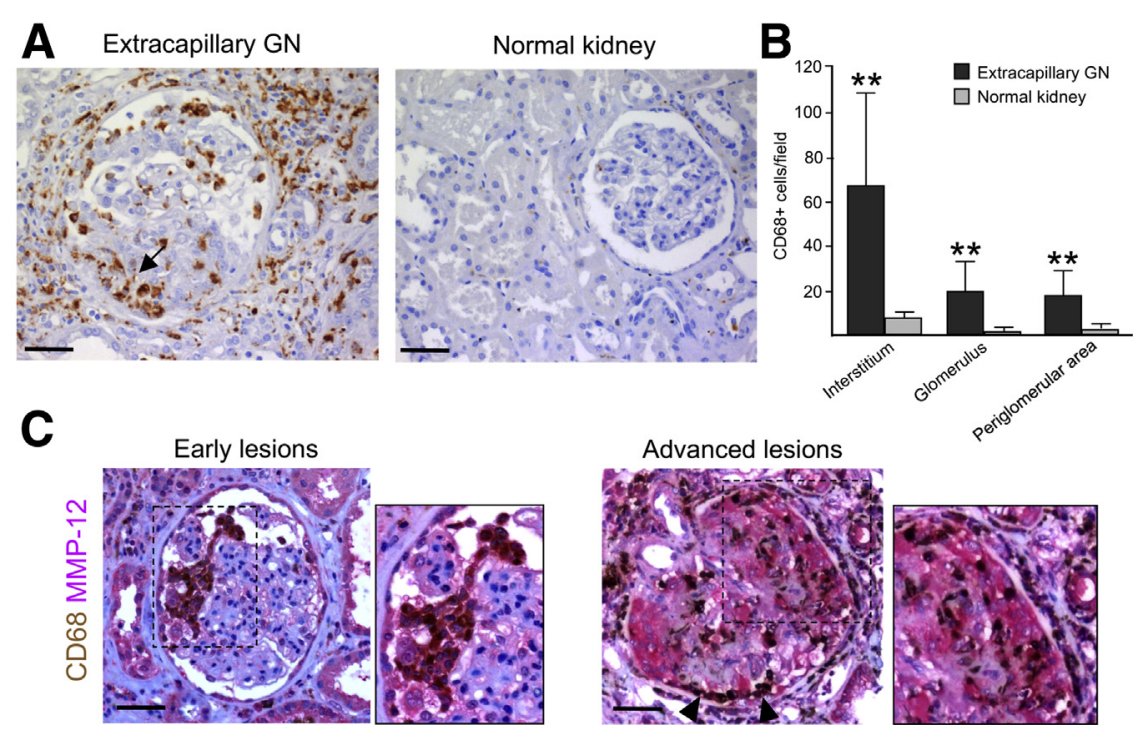

Figure 2 Infiltration of macrophages and matrix metalloproteinase (MMP)-12 accumulation in patients with extracapillary glomerulonephritis (GN). A: Representative immunoperoxidase staining showing a significant increase in $\mathrm{CD} 68^{+}$cells in the glomeruli and interstitium of patients with extracapillary GN compared to age-matched normal kidneys. Arrow shows crescentic lesion. B: Quantitative analysis of $\mathrm{CD}^{+} 8^{+}$macrophages in the interstitium, in the glomerulus, and in the periglomerular area. C: Representative double staining of CD68 (brown signal) and MMP-12 (red signal) in glomeruli of extracapillary GN patients with earlyand advanced-stage histological lesions. Dashed boxes are shown at higher magnification in the insets. Insets show strong MMP-12 expression in the areas where a high number of $\mathrm{CD}^{+} 8^{+}$macrophages are also present. Arrowheads show MMP$12^{+} \mathrm{CD}^{+} 8^{+}$cells along the Bowman capsule. $* * P<0.01$ versus normal kidneys. Scale bars $=50 \mu \mathrm{m}(\mathbf{A}$ and $\mathbf{C})$.

CD68 and MMP-12 double staining, paraffin-embedded kidney sections were processed as described above, and co-incubated with mouse anti-CD68 and rabbit anti-MMP-12 (1:250; Proteintech Group Inc., Chicago, IL) primary antibodies, followed by $\mathrm{MACH} 2$ double stain 2 (Biocare Medical) polymer. Diaminobenzidine and Warp red (Biocare Medical) substrate solutions were used for CD68 and MMP-12, respectively. Slides were finally counterstained with Meyer hematoxylin and observed by light microscopy (ApoTome). Negative controls were obtained by omitting the primary antibodies on adjacent sections. CD68 expression was quantified as the number of positive cells in each glomerulus or in each field $(\times 40)$, at both interstitial and periglomerular areas. MMP-12 and $\mathrm{AT}_{1}$ receptor expression was evaluated in two serial sections for each patient. The positive area for each signal was quantified in all of the glomeruli that were found in kidney biopsy specimens, divided according to the grade of glomerular lesions (early, intermediate, or advanced, up to 20 glomeruli for each grade of lesion). Quantification was performed by using ImageJ version 1.50i (NIH, Bethesda, MD; http://imagej.nih.gov/ij), and values were expressed as a percentage of positive area on total glomerular area.

\section{Immunofluorescence and Confocal Microscopy}

Archived kidney biopsy specimens, snap frozen in liquid nitrogen and embedded in OCT compound, were used for immunofluorescence analysis. Single or double immunostaining for SDF-1, CXCR4, CXCR7, and CD24 expression was performed. Frozen sections ( $2 \mu \mathrm{m}$ thick) were air dried and fixed with cold acetone. For SDF-1 detection, antigen retrieval was performed by boiling sections using a microwave (twice for 5 minutes in $10 \mathrm{mmol} / \mathrm{L}$ citrate buffer, $\mathrm{pH}$ 6.0 , at an operating frequency of $2450 \mathrm{MHz}$ and $600 \mathrm{~W}$ power output). After blocking nonspecific sites with $1 \%$ bovine serum albumin, the following primary antibodies were used: rabbit anti-SDF-1 (1:200; Abcam, Cambridge, UK), rabbit anti-CXCR4 (1:50; Abcam), mouse antiCXCR7 (1:5; R\&D Systems, Minneapolis, MN), and goat anti-CD24 (1:25; Santa Cruz Biotechnology), followed by the specific fluorescein isothiocyanate or Cy3-conjugated secondary antibodies (Jackson Immunoresearch Laboratories, West Grove, PA). Nuclei were stained with DAPI. Negative controls were obtained by omitting primary antibodies on adjacent sections. Fluorescence was examined by an inverted confocal laser scanning microscope (LSM510 Meta; Zeiss). CXCR4 and CXCR7 expression was quantified in all of the glomeruli that were found in patients' biopsy specimens and in normal kidneys (up to 10 glomeruli from each patient), by using ImageJ version 1.50i. Values are expressed as a percentage of positive area in the total glomerular area.

\section{Cell Cultures}

\section{Podocytes}

Conditionally immortalized human podocytes (kindly provided by Prof. M.A. Saleem, Children's Renal Unit and Academic Renal Unit, University of Bristol, Southmead Hospital, Bristol, UK) were cultured under growth-permissive conditions at $33^{\circ} \mathrm{C}$ in RPMI 1640 medium (Invitrogen Corp., Grand Island, NY), as already described. ${ }^{29}$ To induce differentiation, podocytes were seeded on rat collagen type I (BD Bioscience, San Diego, CA) and maintained in nonpermissive conditions at $37^{\circ} \mathrm{C}$ for 12 days.

\section{PEC Isolation}

Human PECs were isolated, as previously described. ${ }^{20,30}$ Briefly, PECs were recovered from total renal cells and isolated from a normal kidney fragment from a young patient (3 years old) who underwent nephrectomy, using CD133- 
immunomagnetic separation. Clones were generated from $\mathrm{CD}_{133^{+}}$cells by limiting dilution, and immunofluorescence and fluorescence-activated cell sorting analysis were used to identify clones positive for CD24 and CD106.

\section{Immunofluorescence Studies in Vitro}

To assess the expression of SDF-1 by podocytes and PECs, cells were incubated for 6 hours with $10^{-7} \mathrm{~mol} / \mathrm{L}$ ang II. Then, cells were fixed in $2 \%$ paraformaldehyde (Electron Microscopy Science) and 4\% sucrose for 15 minutes at $37^{\circ} \mathrm{C}$, permeabilized with Triton $0.3 \%$ for 3 minutes at room temperature, and incubated with blocking solution for 1 hour. Podocytes and PECs were stained with rabbit anti-SDF-1 antibody, followed by a goat antirabbit Cy3-conjugated secondary antibody (Jackson ImmunoResearch Laboratories). Nuclei were counterstained with DAPI. The area occupied by the signal was quantified using ImageJ version $1.50 \mathrm{i}$, and normalized for the number of DAPI-positive cells. Data were expressed as $\operatorname{pixel}^{2} /$ cell.

\section{Migration Assay}

The ability of PECs to migrate toward injured podocytes was assessed in a co-culture transwell system (Costar; Sigma-Aldrich, St. Louis, MO). PECs were seeded in a transwell chamber on the upper side of an 8 - $\mu \mathrm{m}$ permeable membrane, whereas podocytes were co-cultured in the lower chamber. Podocytes and PECs were incubated for 24 hours with endothelial cell growth medium-MV medium (Lonza, Walkersville, MD) plus 2\% fetal bovine serum Hyclone (Hyclone Laboratories, Logan, UT) in the presence or absence of $10^{-7} \mathrm{~mol} / \mathrm{L}$ ang II, $10 \mu \mathrm{g} / \mathrm{mL}$ ang II + SDF-1 neutralizing antibody, or $1 \mu \mathrm{mol} / \mathrm{L}$ ang II + candesartan. At the end of the incubation, PECs at the upper side of the filter were mechanically removed. PECs that migrated to the lower side of the filter were fixed in $11 \%$ glutaraldehyde for 30 minutes at room temperature and then stained with hematoxylin and eosin. The number of cells that had migrated was counted in five to eight random fields for each filter. Data were expressed as the number of transmigrated PECs/high-power field (magnification, $\times 10$ ).

\section{Statistical Analysis}

Results were expressed as means $\pm \mathrm{SD}$. The $t$-test was applied for quantification of Bowman capsule rupture and CD68, CXCR4, and CXCR7 expression. Statistical analysis of MMP-12 and $\mathrm{AT}_{1}$ receptor expression was performed using analysis of variance with Tukey post hoc analysis for multiple comparisons; for in vitro experiments, comparisons were made using the Bonferroni post hoc test. Statistical significance was defined as $P<0.05$.

\section{Results}

\section{Patient Population}

Ten patients with extracapillary lesions (one patient affected by anti-GBM GN, four by antineutrophil cytoplasm antibody-associated vasculitis, and five by immune complex-mediated GN) were enrolled in the study. Clinical and histopathological characteristics of patients at the time of the biopsy are outlined in Table 1. Their mean age was $53.4 \pm 20.9$ years. Eight patients had renal impairment at onset, whereas two had initial normal serum creatinine levels, despite active sediment. All but one completely anuric patient had a high degree of proteinuria (nephrotic in four cases) and all had hematuria (one with macrohematuria). In terms of histology, most glomeruli had extracapillary lesions, whose index averaged 2.0, and a large number of the glomeruli exhibited global or segmental sclerosis.

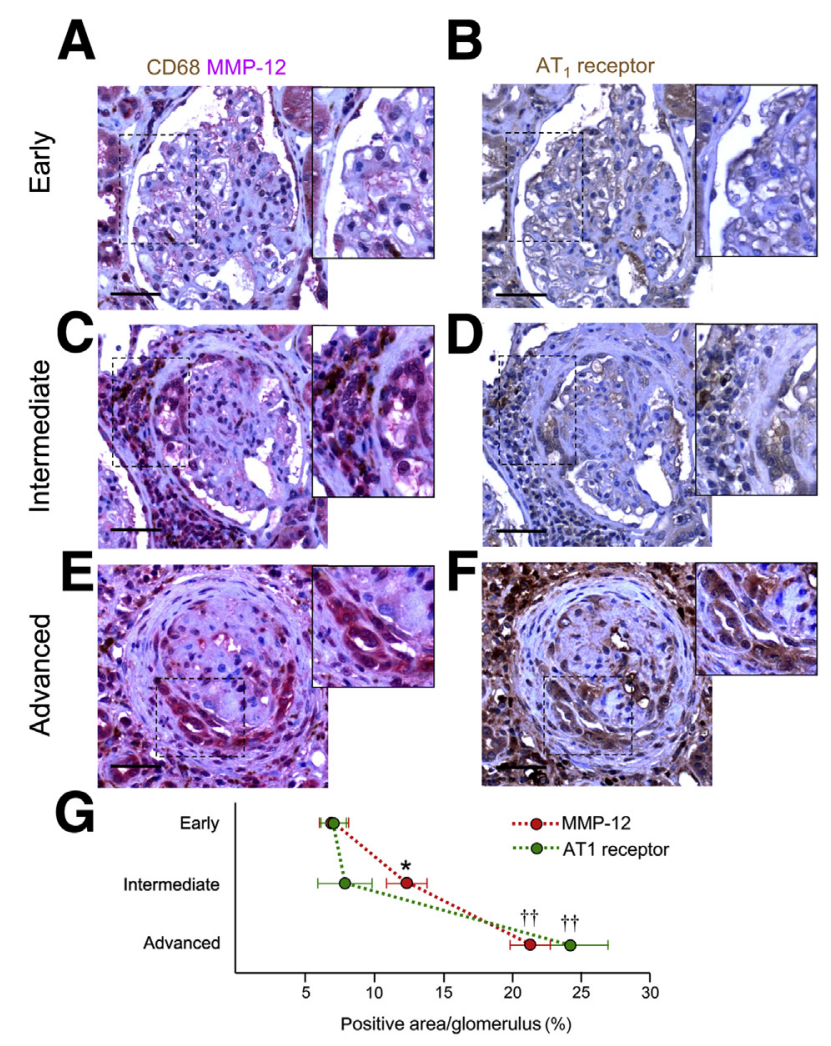

Figure 3 Macrophage and matrix metalloproteinase (MMP)-12 accumulation is an early event in the development of crescentic lesions compared to angiotensin II type 1 receptor ( $\mathrm{AT}_{1}$ receptor) up-regulation. A-F: Representative images of serial sections labeled with CD68 (brown) and MMP-12 (red; $\mathbf{A}, \mathbf{C}$, and $\mathbf{E}$ ), and $\mathrm{AT}_{1}$ receptor (brown; $\mathbf{B}, \mathbf{D}$, and $\mathbf{F}$ ), showing glomeruli with early-stage (A and $\mathbf{B})$, intermediate-stage (C and D), and advanced-stage (E and $\mathbf{F}$ ) lesions in patients with extracapillary glomerulonephritis. Dashed boxes are shown at higher magnification in the insets. Insets show areas with different degrees of glomerular lesions and protein expression. G: Quantitative analysis of MMP-12 and $\mathrm{AT}_{1}$ receptor expression in the glomerular area, in patients' glomeruli with early-, intermediate-, and advanced-stage lesions. ${ }^{*} P<0.05$ versus glomeruli with early-stage lesions; ${ }^{\dagger \dagger} P<0.01$ versus glomeruli with intermediatestage lesions. Scale bars $=50 \mu \mathrm{m}(\mathbf{A}-\mathbf{F})$. 

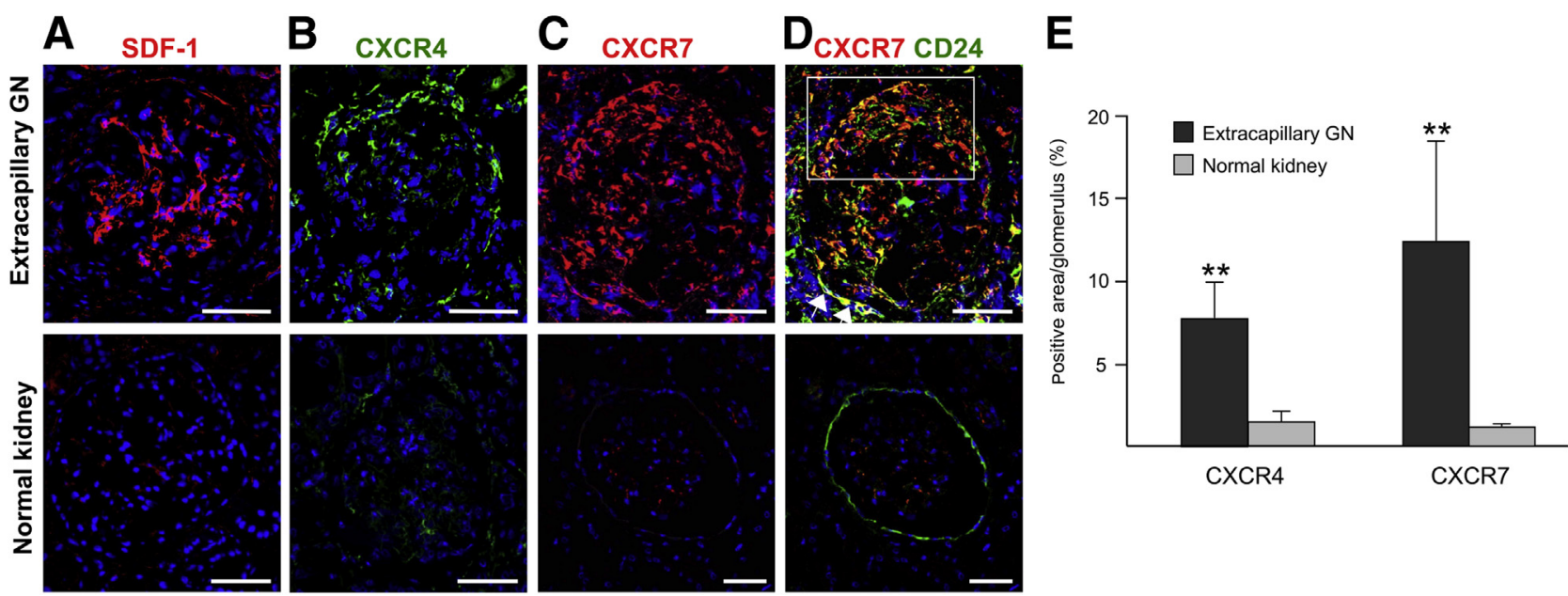

Figure 4 Stromal cell-derived factor-1 (SDF-1) and C-X-C chemokine receptor (CXCR) 7 are up-regulated in patients with extracapillary glomerulonephritis (GN). A: Representative immunofluorescence staining for SDF-1 (red) showing increased glomerular expression in patients with extracapillary GN compared to age-matched normal kidney. B: The expression of the SDF-1 receptor CXCR4 (green) is up-regulated in patients with extracapillary GN as compared with agematched normal kidney. C: SDF-1 receptor CXCR7 (red) is highly expressed in patients with extracapillary GN as compared with age-matched normal kidney. D: CXCR7 (red) colocalizes with progenitor cell marker CD24 (green) in patients with extracapillary GN, in particular in areas with crescentic lesions (boxed area) or along the Bowman capsule (arrows). CD24 ${ }^{+}$cells do not coexpress CXCR7 in age-matched normal kidney. DAPI (blue) stained nuclei. E: Quantitative analysis of CXCR4 and CXCR7 expression in the glomerular area. ${ }^{*} P<0.01$ versus normal kidneys. Scale bars $=50 \mu \mathrm{m}(\mathbf{A}-\mathbf{D})$.

\section{Alterations of Bowman Capsule and GBM in Patients with Extracapillary GN}

Most glomeruli $(59.25 \% \pm 19.50 \%)$ in our patient population showed recurring breaks along the Bowman capsule, which were heterogeneous in size (score, 0 to 4: $0.94 \pm 0.67$ in patients with extracapillary GN versus $0.00 \pm 0.00$ in normal kidneys; $P<0.01$ ) (Figure 1A). Several segmental irregularities and wrinkling were also visible along the GBM, with areas of disrupted continuity (Figure 1A). Conversely, glomeruli from normal kidneys displayed a linear GBM without evident signs of rupture or irregularities (Figure 1B). Through transmission electron microscopy analysis of affected kidney sections, GBM ruptures (Figure 1C) as well as GBM discontinuities and gaps (Figure 1, D-H) were detected, in association with red blood cells that had spilled from the glomerular capillaries, and were consequently found on the outer side of the glomerular filtration barrier (Figure 1D).

\section{$\mathrm{CD}^{+} 8^{+}$Macrophage Accumulation Already 0ccurs in Early-Stage Crescentic Lesions}

Compared to normal kidneys, there was a significant increase in $\mathrm{CD} 8^{+}$macrophages in the interstitium, glomerulus, and periglomerular area in patients with extracapillary GN $(P<0.01)$ (Figure 2, A and B). At the glomerular level, $\mathrm{CD} 8^{+}$cells were mainly localized in crescentic lesions (Figure 2A). In patients with extracapillary GN, the high infiltration of $\mathrm{CD}^{+} 8^{+}$macrophages was associated with increased expression of their own elastase MMP-12, an extracellular matrix-degrading enzyme that is capable of degrading almost all of the components of glomerular membranes $^{31,32}$ (Figure 2C). Double staining with CD68 and MMP-12 revealed that the accumulation of macrophages within the glomeruli was closely correlated with the release of macrophage elastase, paralleling the progression of the hyperplasia (Figure 2C). In particular, in glomeruli with lesions at early stages, $\mathrm{CD} 68^{+} \mathrm{MMP}-12^{+}$cells were present only along the GBM in focal areas of the glomerular tuft. In contrast, in glomeruli affected by advanced lesions, CD68 ${ }^{+}$MMP- $12^{+}$cells were abundant both outside and inside the glomerulus, and accumulated mainly in crescents and along the Bowman capsule.

\section{$\mathrm{AT}_{1}$ Receptor Expression Is Up-Regulated Only in Advanced-Stage Crescentic Lesions}

All glomeruli that were found in patients' biopsy specimens were divided according to the grade of lesions: those showing negligible proliferative lesions (Figure 3, A and B), those displaying intermediate lesions with synechiae and segmental extracapillary proliferation (Figure 3, C and D), and glomeruli with severe crescents (Figure 3, E and F). CD68, MMP-12, and $\mathrm{AT}_{1}$ receptor staining, performed in two consecutive kidney sections, were detected faintly in glomeruli with negligible/early proliferative lesions (Figure 3, A, B, and G). In glomeruli with segmental/intermediate extracapillary proliferation, macrophage infiltration and their metalloproteinase increased in the periglomerular and glomerular area $(P<0.05)$ (Figure 3, C and G), whereas $\mathrm{AT}_{1}$ receptor expression was still faint at this stage of the disease (Figure 3, D and G). The expression of $\mathrm{AT}_{1}$ receptor increased abundantly in glomeruli displaying advanced crescentic lesions, in particular in areas of 


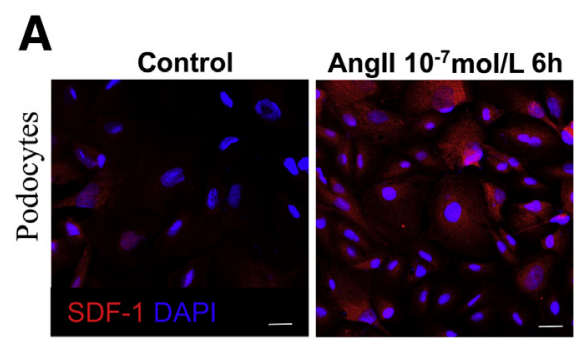

B
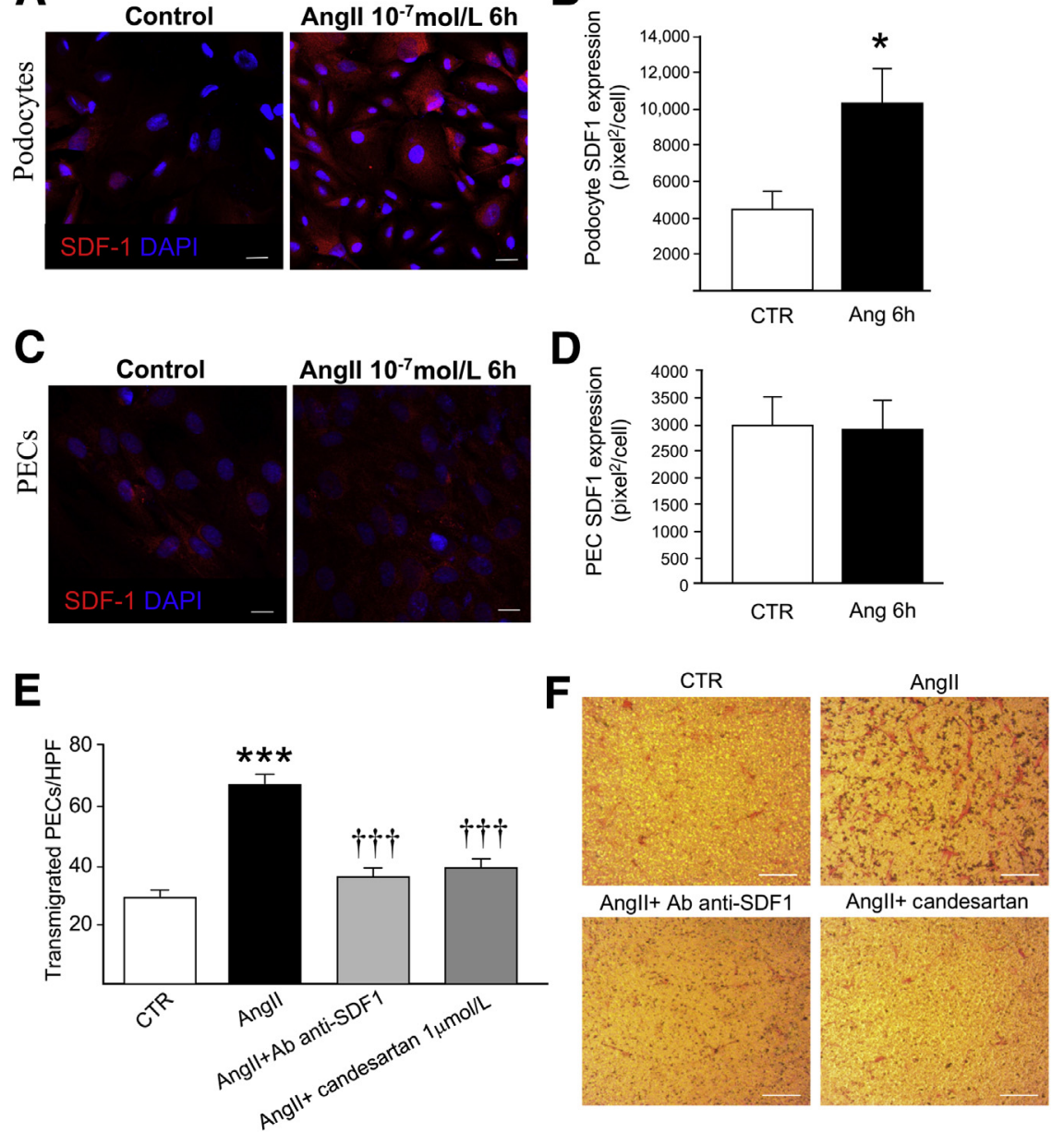

Figure 5 Stromal cell-derived factor-1 (SDF1) is produced by podocytes, but not by parietal epithelial cells (PECS), after stimulation with angiotensin II (Ang II), and plays a crucial role in PEC dysfunction. A: Representative immunofluorescence staining for SDF-1 (red) in human podocytes before and after ang II stimulation. DAPI (blue) stained nuclei. B: Quantification of SDF-1 staining in human podocytes showing an increase in chemokine expression after 6-hour stimulation with ang II. C: SDF-1 expression (red) in human PECs before and after ang II stimulation. DAPI (blue) stained nuclei. D: Histogram shows that SDF-1 expression does not increase after stimulation with ang II. E: Quantification of PEC migration at 24 hours in co-culture transwells from the upper chamber toward unstimulated or ang II-activated podocytes in the presence or absence of the anti-SDF-1 antibody or candesartan. Data were expressed as number of PECs that migrated from the upper chamber across the filter toward podocytes/highpower field. F: Representative images of transmigrated PECs stained with hematoxylin and eosin, toward untreated [control (CTR)], ang II-activated podocytes, ang II + antibody (Ab) anti-SDF-1, or ang II + candesartan treated podocytes. ${ }^{*} P<0.05,{ }^{* * *} P<0.001$ versus CTR; ${ }^{\dagger \dagger} P<0.001$ versus ang II. Scale bars: $20 \mu \mathrm{m}(\mathbf{A})$; $50 \mu \mathrm{m}$ (C); $100 \mu \mathrm{m}$ (F). hyperplasia in which MMP-12 was also detected $(P<0.01)$ (Figure 3, E-G).

\section{SDF-1 and Its Receptors CXCR4 and CXCR7 Are Up- Regulated in Biopsy Specimens of Patients with Advanced Crescentic Lesions}

Given the contribution of the immune-related chemokine SDF-1 and its receptor CXCR4 in proliferative diseases, ${ }^{22}$ herein we studied in depth the role of SDF-1 in mediating progenitor cell activation by evaluating the expression of the alternative SDF-1 receptor CXCR7, in biopsy specimens of patients with extracapillary GN. A previous study has shown that CXCR7 receptor is a critical mediator of the therapeutic homing of human PECs/ progenitor cells in mouse injured renal tissue. ${ }^{33}$ However, its expression in human biopsy specimens from patients with extracapillary lesions has not been reported yet. In line with our previous data, ${ }^{22}$ glomeruli with advanced crescentic lesions showed increased expression of the chemokine SDF-1 and of its receptor CXCR4 compared to normal kidneys (Figure 4, A and B). Concomitantly with CXCR4 receptor up-regulation $(P<0.01)$ (Figure 4, B and E), in the same biopsy specimens, increased expression of CXCR7 was observed $(P<0.01)$
(Figure 4, C and E). Double staining with CXCR7 and the progenitor cell marker CD24 revealed that SDF-1 receptor CXCR7 was mainly expressed by renal progenitors in the Bowman capsule and within crescentic lesions (Figure 4D), similar to the pattern previously observed for CXCR4. ${ }^{22}$

\section{Ang II Promotes SDF-1 Production by Cultured Podocytes}

To further confirm the cellular source of SDF-1 production observed in biopsy specimens of patients with extracapillary GN, and to analyze whether ang II was involved in SDF-1 stimulation, cultured podocytes and PECs/progenitor cells were exposed to $10^{-7} \mathrm{~mol} / \mathrm{L}$ ang II for 6 hours and subjected to immunofluorescence analysis. A faint SDF-1 signal was observed in podocytes and PECs under basal conditions (Figure 5, A and C). Exposure to ang II significantly increased $(P<0.05)$ SDF-1 expression in podocytes (Figure 5, A and B), whereas the peptide failed to induce SDF-1 overexpression in cultured PECs, indicating the inability of these cells to produce the chemokine (Figure 5, C and D). These results confirmed data obtained from kidney biopsy specimens (Figure 4A). ${ }^{22}$ 


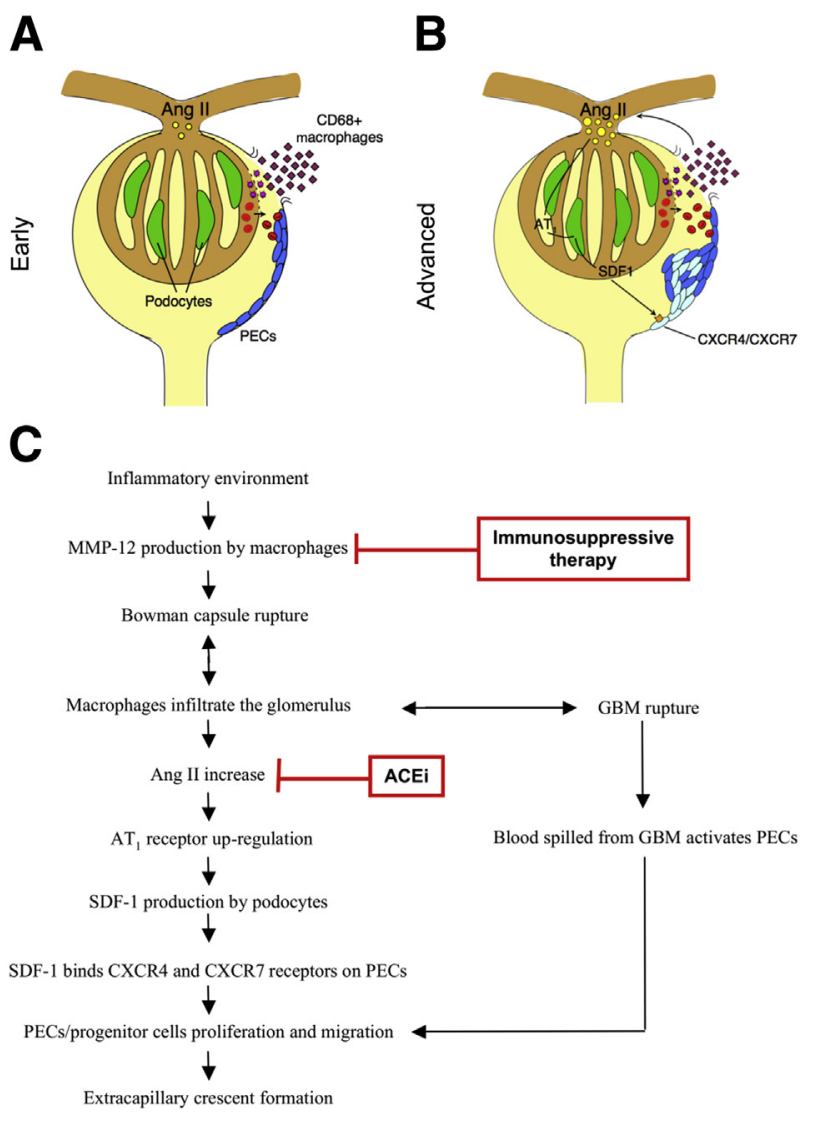

Figure 6 A: The early phase of the disease is characterized by $\mathrm{CD}_{68}{ }^{+}$ macrophages (violet), which by releasing matrix metalloproteinase (MMP)12 (pink) induce ruptures in Bowman capsule and glomerular basement membrane (GBM). Gaps in GBM allow red blood cells (red) to exit the glomerular capillaries and to reach parietal epithelial cells (PECs; blue). Activated PECs begin to proliferate. B: The late phase of the disease is characterized by macrophage-induced increase in angiotensin II (ang II; yellow), which via angiotensin II type 1 receptor $\left(\mathrm{AT}_{1}\right.$ receptor) activates podocytes (green) to produce the stromal cell-derived factor-1 (SDF-1) chemokine. SDF-1 binds C-X-C chemokine receptor (CXCR) 4 and CXCR7 on $\mathrm{PEC}$, promoting their proliferation and migration toward the glomerular tuft, resulting in crescent formation. C: Schematic representation of the mechanisms involved in crescentic lesion formation in patients with extracapillary glomerulonephritis. ACEi, angiotensin-converting enzyme inhibitor.

\section{Ang II Induces PEC/Progenitor Cell Transmigration via SDF-1 and $\mathrm{AT}_{1}$ Receptor}

To explore in depth the mechanisms through which ang II induced PEC activation, we studied PEC/progenitor cell migration using a transwell system, where a porous membrane physically separated PECs and podocytes, treated or not treated with ang II. The migration of PECs from the upper chamber across the membrane toward ang II-activated podocytes was significantly higher than that observed toward untreated podocytes (Figure 5, E and F). The functional blockade of podocyte-derived SDF-1 with a specific antibody significantly reduced PEC transmigration toward activated podocytes (Figure 5, E and F). Similarly, treatment with the $\mathrm{AT}_{1}$ receptor antagonist candesartan significantly limited the number of
PECs that migrated toward ang II-treated podocytes (Figure 5, E and F).

\section{Discussion}

This study sought to describe in depth the sequential events and molecular interactions that occur during the onset and evolution of crescentic lesions. Serial section immunohistochemical analysis was performed on kidney biopsy specimens of rapidly progressive GN patients, followed by rigorously designed in vitro assays. These approaches allowed us to draw a step-by-step sequence from early abnormalities to aberrant PEC/progenitor cell proliferation and migration toward the glomerular tuft, and to suggest which mediators may be involved.

We have observed that macrophages, possibly recruited by neutrophils, ${ }^{34}$ accumulated in the interstitium, in the periglomerular area, and in the tuft since the earliest stages of glomerular injury, and their infiltration progressively increased inside the glomeruli, paralleling the progression of hyperplasia. Interstitial macrophages produced a growing amount of MMP-12 elastase, which, coherently with data obtained in a rat model of anti-GBM GN, ${ }^{32}$ was already expressed before the onset of severe glomerular lesions. The proteolytic enzyme MMP-12 may contribute to focal ruptures of Bowman capsule, which are typically found in patients with extracapillary $\mathrm{GN},{ }^{2}$ and have been confirmed in our patients through transmission electron microscopy observations. Interestingly, red blood cells found on the outer side of the glomerular capillaries in correspondence with GBM discontinuities or breaks are evidence of an abnormal leakage of blood from glomerular capillaries, which is probably the result of MMP-12-driven glomerular membrane lysis, and which clinically translates into patients experiencing hematuria. ${ }^{2}$ It has been shown that exposure of cultured human PECs to blood serum promotes their activation and the acquisition of a proliferative and mitogenic phenotype, suggesting a possible relationship between blood leakage from the glomerular capillaries and crescent formation. ${ }^{35}$ Thus, our in vivo observations, combined with previous in vitro findings, ${ }^{35}$ can explain how, in patients with extracapillary GN, macrophage-driven lysis of glomerular membranes could lead to the contact between blood and PECs/progenitor cells, which, in turn, begin to proliferate, possibly contributing to crescent formation.

On the basis of our previous data showing that renal progenitors of the Bowman capsule constitutively express $\mathrm{AT}_{1}$ receptor, the expression of which was markedly augmented in crescentic lesions of experimental and human extracapillary $\mathrm{GN},{ }^{22}$ herein we perform an unprecedented analysis to investigate the sequence of events linking the infiltration of inflammatory cells to the activation of the renin-angiotensin system during the evolution of crescentic lesions. Although MMP-12 produced by macrophages was expressed in the early stage of glomerular injury, $\mathrm{AT}_{1}$ receptor, presumably expressed by 
progenitor cells, ${ }^{22}$ could only be detected in advanced lesions, suggesting that the involvement of the renin-angiotensin system was a later phenomenon in the development of crescents with regard to MMP-12-expressing macrophage infiltration. Notably, $\mathrm{AT}_{1}$ receptor was localized in glomerular areas in which MMP-12 was detected, providing new insights into the possible role of infiltrating macrophages in promoting PEC proliferation and migration. Thus, it is conceivable that crescent formation is primarily promoted by infiltrating macrophages, which may initially induce capillary blood leakage through MMP-12-driven GBM lysis, and, once accumulated in the glomerulus, are activated to produce ang II. ${ }^{36}$ Ang II proinflammatory activities via $\mathrm{AT}_{1}$ receptor ${ }^{37}$ can, in turn, further trigger phlogogenic cell infiltration, ultimately resulting in a vicious cycle that worsens disease progression.

In vitro experiments then provided further clarification regarding the role of ang II and SDF-1 in regulating podocyte and PEC cross talk during hyperplastic lesion onset and evolution. Podocytes were the only glomerular source of SDF-1, whose production was directly stimulated by ang II through $\mathrm{AT}_{1}$ receptor. Moreover, podocyte-produced SDF-1 played a pivotal role in mediating ang II-dependent PEC activation. These observations further corroborated and explained in vivo findings showing increased expression of SDF-1 in patients' podocytes and the relative receptors CXCR4 and CXCR7 in parietal progenitor cells and in crescentic lesions. To the best of our knowledge, this is the first piece of evidence showing how the ang II/AT $\mathrm{A}_{1}$ receptor/ SDF-1 pathway orchestrates the pathological cross talk between podocytes and PECs in proliferative diseases.

In summary, these data indicate that PEC proliferation and migration in extracapillary GN can conceivably be triggered by multiple independent, but interconnected, pathological pathways. On one hand, macrophages may induce PEC activation by promoting blood spillage through the GBM, and producing ang II, which via $\mathrm{AT}_{1}$ receptor stimulates podocytes to express SDF-1. On the other hand, excessive production of ang II stimulates podocytes to produce SDF-1, in turn promoting parietal progenitor activation via CXCR4 and CXCR7 receptors (Figure 6).

The understanding of the cross talk between these pathways, which have never been described and characterized in detail, provides crucial new insights into the pathogenesis of extracapillary GN. The present findings help to explain a previous preliminary study in rats and in a patient with advanced nephropathy, showing that ACE inhibitor treatment limits PEC activation and reduces the frequency and extension of crescents, paralleling the normalization of SDF-1/CXCR4 expression. ${ }^{19,21,22}$

Thus, we provide a rationale for future studies that aim to investigate whether ACE inhibitor therapy given on top of immunosuppression may be able to limit or prevent crescent formation in extracapillary GN more effectively than immunosuppressive therapy alone. Treatment with ACE inhibitor may help to inhibit both the vicious cycle of macrophage infiltration and the ang II-dependent SDF-1 pathway, eventually translating into accelerated renal function recovery and, conceivably, reducing the need for immunosuppressive therapy and the related serious adverse events.

\section{Acknowledgments}

We thank Dr. Mauro Abbate for helpful discussions, Kerstin Mierke for English language editing, and Prof. M.A. Saleem (Children's Renal Unit and Academic Renal Unit, University of Bristol, Southmead Hospital, Bristol, UK) for conditionally immortalized human podocytes.

\section{References}

1. Couser WG: Rapidly progressive glomerulonephritis: classification, pathogenetic mechanisms, and therapy. Am J Kidney Dis 1988, 11: 449-464

2. Jennette JC: Rapidly progressive crescentic glomerulonephritis. Kidney Int 2003, 63:1164-1177

3. Primary glomerular disease. Brenner \& Rector's The Kidney, ed 9. Edited by Taal MW, Chertow GM, Marsden PA, Skorecki K, Yu ASL, Brenner BM. Philadelphia PA: Elsevier Saunders, 2012. pp. 1153-1168

4. Cattell V, Jamieson SW: The origin of glomerular crescents in experimental nephrotoxic serum nephritis in the rabbit. Lab Invest 1978, 39:584-590

5. Atkins RC, Holdsworth SR, Glasgow EF, Matthews FE: The macrophagen in human rapidly progressive glomerulonephritis. Lancet 1976, $1: 830-832$

6. Boucher A, Droz D, Adafer E, Noel LH: Relationship between the integrity of Bowman's capsule and the composition of cellular crescents in human crescentic glomerulonephritis. Lab Invest 1987, 56: $526-533$

7. Nitta K, Horita S, Honda K, Uchida K, Watanabe T, Nihei H, Nagata M: Glomerular expression of cell-cycle-regulatory proteins in human crescentic glomerulonephritis. Virchows Arch 1999, 435:422-427

8. Jennette JC, Hipp CG: The epithelial antigen phenotype of glomerular crescent cells. Am J Clin Pathol 1986, 86:274-280

9. Lan HY, Nikolic-Paterson DJ, Atkins RC: Involvement of activated periglomerular leukocytes in the rupture of Bowman's capsule and glomerular crescent progression in experimental glomerulonephritis. Lab Invest 1992, 67:743-751

10. Ophascharoensuk V, Pippin JW, Gordon KL, Shankland SJ, Couser WG, Johnson RJ: Role of intrinsic renal cells versus infiltrating cells in glomerular crescent formation. Kidney Int 1998, 54:416-425

11. Moeller MJ, Soofi A, Hartmann I, Le Hir M, Wiggins R, Kriz W, Holzman LB: Podocytes populate cellular crescents in a murine model of inflammatory glomerulonephritis. J Am Soc Nephrol 2004, 15: $61-67$

12. Bariety J, Bruneval P, Meyrier A, Mandet C, Hill G, Jacquot C: Podocyte involvement in human immune crescentic glomerulonephritis. Kidney Int 2005, 68:1109-1119

13. Thorner PS, Ho M, Eremina V, Sado Y, Quaggin S: Podocytes contribute to the formation of glomerular crescents. J Am Soc Nephrol 2008, 19:495-502

14. Ding M, Cui S, Li C, Jothy S, Haase V, Steer BM, Marsden PA, Pippin J, Shankland S, Rastaldi MP, Cohen CD, Kretzler M, Quaggin SE: Loss of the tumor suppressor Vhlh leads to upregulation of Cxcr4 and rapidly progressive glomerulonephritis in mice. Nat Med 2006, 12:1081-1087

15. Smeets B, Angelotti ML, Rizzo P, Dijkman H, Lazzeri E, Mooren F, Ballerini L, Parente E, Sagrinati C, Mazzinghi B, Ronconi E, Becherucci F, Benigni A, Steenbergen E, Lasagni L, Remuzzi G, 
Wetzels J, Romagnani P: Renal progenitor cells contribute to hyperplastic lesions of podocytopathies and crescentic glomerulonephritis. J Am Soc Nephrol 2009, 20:2593-2603

16. Mundel P, Shankland SJ: Glomerular podocytes and adhesive interaction with glomerular basement membrane. Exp Nephrol 1999, 7: $160-166$

17. Smeets B, Uhlig S, Fuss A, Mooren F, Wetzels JF, Floege J, Moeller MJ: Tracing the origin of glomerular extracapillary lesions from parietal epithelial cells. J Am Soc Nephrol 2009, 20:2604-2615

18. Shankland SJ, Smeets B, Pippin JW, Moeller MJ: The emergence of the glomerular parietal epithelial cell. Nat Rev Nephrol 2014, 10: $158-173$

19. Rizzo P, Novelli R, Benigni A, Remuzzi G: Inhibiting angiotensinconverting enzyme promotes renal repair by modulating progenitor cell activation. Pharmacol Res 2016, 108:16-22

20. Angelotti ML, Ronconi E, Ballerini L, Peired A, Mazzinghi B, Sagrinati C, Parente E, Gacci M, Carini M, Rotondi M, Fogo AB, Lazzeri E, Lasagni L, Romagnani P: Characterization of renal progenitors committed toward tubular lineage and their regenerative potential in renal tubular injury. Stem Cells 2012, 30:1714-1725

21. Benigni A, Morigi M, Rizzo P, Gagliardini E, Rota C, Abbate M, Ghezzi S, Remuzzi A, Remuzzi G: Inhibiting angiotensin-converting enzyme promotes renal repair by limiting progenitor cell proliferation and restoring the glomerular architecture. Am J Pathol 2011, 179: 628-638

22. Rizzo P, Perico N, Gagliardini E, Novelli R, Alison MR, Remuzzi G, Benigni A: Nature and mediators of parietal epithelial cell activation in glomerulonephritides of human and rat. Am J Pathol 2013, 183: $1769-1778$

23. Eng DG, Sunseri MW, Kaverina NV, Roeder SS, Pippin JW, Shankland SJ: Glomerular parietal epithelial cells contribute to adult podocyte regeneration in experimental focal segmental glomerulosclerosis. Kidney Int 2015, 88:999-1012

24. Lasagni L, Angelotti ML, Ronconi E, Lombardi D, Nardi S, Peired A, Becherucci F, Mazzinghi B, Sisti A, Romoli S, Burger A, Schaefer B, Buccoliero A, Lazzeri E, Romagnani P: Podocyte regeneration driven by renal progenitors determines glomerular disease remission and can be pharmacologically enhanced. Stem Cell Rep 2015, 5:248-263

25. Pippin JW, Sparks MA, Glenn ST, Buitrago S, Coffman TM, Duffield JS, Gross KW, Shankland SJ: Cells of renin lineage are progenitors of podocytes and parietal epithelial cells in experimental glomerular disease. Am J Pathol 2013, 183:542-557

26. Lapidot T, Dar A, Kollet O: How do stem cells find their way home? Blood 2005, 106:1901-1910
27. Ratajczak MZ, Zuba-Surma E, Kucia M, Reca R, Wojakowski W, Ratajczak J: The pleiotropic effects of the SDF-1-CXCR4 axis in organogenesis, regeneration and tumorigenesis. Leukemia 2006, 20: 1915-1924

28. Kim S, Iwao H: Molecular and cellular mechanisms of angiotensin IImediated cardiovascular and renal diseases. Pharmacol Rev 2000, 52 : $11-34$

29. Gagliardini E, Perico N, Rizzo P, Buelli S, Longaretti L, Perico L, Tomasoni S, Zoja C, Macconi D, Morigi M, Remuzzi G, Benigni A: Angiotensin II contributes to diabetic renal dysfunction in rodents and humans via Notch1/Snail pathway. Am J Pathol 2013, 183:119-130

30. Morigi M, Locatelli M, Rota C, Buelli S, Corna D, Rizzo P, Abbate M, Conti D, Perico L, Longaretti L, Benigni A, Zoja C, Remuzzi G: A previously unrecognized role of $\mathrm{C} 3 \mathrm{a}$ in proteinuric progressive nephropathy. Sci Rep 2016, 6:28445

31. Nenan S, Boichot E, Lagente V, Bertrand CP: Macrophage elastase (MMP-12): a pro-inflammatory mediator? Mem Inst Oswaldo Cruz 2005, 100(Suppl 1):167-172

32. Kaneko Y, Sakatsume M, Xie Y, Kuroda T, Igashima M, Narita I, Gejyo F: Macrophage metalloelastase as a major factor for glomerular injury in anti-glomerular basement membrane nephritis. J Immunol 2003, 170:3377-3385

33. Mazzinghi B, Ronconi E, Lazzeri E, Sagrinati C, Ballerini L, Angelotti ML, Parente E, Mancina R, Netti GS, Becherucci F, Gacci M, Carini M, Gesualdo L, Rotondi M, Maggi E, Lasagni L, Serio M, Romagnani S, Romagnani P: Essential but differential role for CXCR4 and CXCR7 in the therapeutic homing of human renal progenitor cells. J Exp Med 2008, 205:479-490

34. Mayadas TN, Rosetti F, Ernandez T, Sethi S: Neutrophils: game changers in glomerulonephritis? Trends Mol Med 2010, 16: 368-378

35. Ryu M, Migliorini A, Miosge $\mathrm{N}$, Gross $\mathrm{O}$, Shankland $\mathrm{S}$, Brinkkoetter PT, Hagmann H, Romagnani P, Liapis H, Anders HJ: Plasma leakage through glomerular basement membrane ruptures triggers the proliferation of parietal epithelial cells and crescent formation in non-inflammatory glomerular injury. J Pathol 2012, 228: 482-494

36. Yanagitani Y, Rakugi H, Okamura A, Moriguchi K, Takiuchi S, Ohishi M, Suzuki K, Higaki J, Ogihara T: Angiotensin II type 1 receptor-mediated peroxide production in human macrophages. Hypertension 1999, 33:335-339

37. Brasier AR, Recinos A 3rd, Eledrisi MS: Vascular inflammation and the renin-angiotensin system. Arterioscler Thromb Vasc Biol 2002, 22 : $1257-1266$ 